\title{
Activation thresholds and operating characteristics of commercial alarm products to provide surveillance for dementia caregivers
}

\author{
Shawn P. Applegarth $M^{\boxplus S M E}{ }^{a}$ \\ Meredeth Rowe PhD, RN, FAAN, FGSA ${ }^{b}$, \\ William Kearns $\mathrm{PhD}^{\mathrm{c}}$ \\ Mary E. Bowen PhD \\ E: mary.bowen3@va.gov
}

\begin{abstract}
aames A. Haley Veterans Hospital, HSR\&D/RR\&D Center of Excellence, Tampa, Florida, USA; ${ }^{b}$ College of Nursing, The University of South Florida, Tampa, Florida, USA; 'Department of Rehabilitation and Mental Health Counseling, The University of South Florida, Tampa, Florida, USA

S.P. Applegarth ${ }^{\dagger}$, M. Rowe, W. Kearns, M.E. Bowen. Activation thresholds and operating characteristics of commercial alarm products to provide surveillance for dementia caregivers. Gerontechnology 2013;11(3):480-487; doi:10.4017/gt.2013.11.3.007.00 Objective A laboratory evaluation was conducted on selected commercial technologies designed to prevent or manage dementia-related missing incidents from the home. Methods Fourteen products representing four unique categories (Pressure Activated Systems, Pull Tab Alarms, Audible Alarms, and Optically Activated Alarms) of behavioral management were evaluated under controlled laboratory conditions for: sound pressure levels $(\mathrm{dB})$, power consumption (watts), battery life, and alarm frequency (pitch). Other variables such as activation force and monitoring/tracking capabilities were tested where applicable to the device category. Results There was wide performance variability regardless of device category, indicating that the devices may fail to successfully capture a missing incident. Implications The development of standardized protocols for testing devices intended for use in the home setting coupled with a broader evaluation of existing technologies would provide a more complete understanding of which products may manage or inhibit missing incidents in persons with dementia.
\end{abstract}

\section{Keywords: executive function deficits, wayfinding, surveillance, wandering}

Most persons with dementia live in the home setting and rely on care from informal caregivers, usually relatives. Often there is a single caregiver, such as a spouse, who is responsible for providing a safe environment 24 hours a day. While most persons with dementia and their caregivers want to continue to provide home-centered care and allow as much independence as possible, this is a significant challenge given that the deficits associated with dementia lead to errors that put the individual in dangerous situations. For instance, when the caregiver and person with dementia are in separate areas of the home, the person with dementia may mistakenly leave the home in a misdirected attempt to find the caregiver. Deficits such as loss of executive function and judgmental errors compound the initial error preventing the individual from undertaking a simple correction to turn around and return to the home. Once lost in the community, the individual is at risk of death ${ }^{1}$.

Missing incidents are relatively common. For example, the prevalence of a missing incident from the home or out in the community is about 0.65/ year among veterans with dementia ${ }^{2}$. Missing incidents are associated with increased caregiver stress, burden ${ }^{3,4}$, high health care costs, institutionalization, and death ${ }^{5}$. Most missing incidents occur from the home with no noted mental status changes or changes in routine and caregivers report seeing the person with dementia about 10 minutes prior to the missing incident ${ }^{2}$. Given that most persons with dementia are missing from the home and these incidents are largely unpredictable, finding technologies that can assist the caregiver with ongoing surveillance in the home to prevent or manage a missing incident would be beneficial.

Some missing incidents occur at night when persons with dementia awaken and leave the home unattended ${ }^{1}$. Dementia-related alterations in sleep-wake patterns may result in increased night awakenings or early morning awakenings. Thus, during the time the caregiver is getting needed rest, the person with dementia is getting up and moving around. Approximately 15\% of 
unattended home exits occur during the evening and night ${ }^{2}$, as well as approximately $38 \%$ of all injuries that occur at home ${ }^{6}$. So the caregiver must attempt to 'sleep with an open ear' and provide needed supervision throughout the 24hour period. This can result in fatigue, which is another factor in the decision to place persons with dementia in a formal care setting.

Technology has been used in many applications to provide surveillance and more specific technologies have been developed to assist caregivers in providing surveillance. While there has been much academic discussion and research on the need for technologies and systems to help older adults age in place, there are relatively few commercial technologies available for use by a caregiver. Of these technologies, most use a magnetic tether, infrared light, or pressure for location sensing. These products are safe and do not interfere with the home environment. However, as implemented in commercial products it remains unclear whether or not these technologies are practical, useful, reliable, and dependable for caregivers of persons with dementia. A systematic investigation of these technologies is needed to determine how well these products may work in homes of persons with dementia and how well these technologies may be used to prevent a missing incident. Thus, the purpose of this study was to systematically evaluate existing technologies that are marketed as products to assist caregivers with surveillance or improving the safety of the home.

This paper presents a laboratory-based evaluation of commercially available products that can be used in home settings to prevent or manage missing incidents.

\section{Methodology \\ Selection of products}

Prior to beginning the evaluation, a comprehensive search of commercially available products was conducted to determine general product categories and the feasibility of objectively testing each product. A description of the device categories, which can be used to monitor wandering (for instance, random, lapping, pacing patterns) or missing incidents from the home ${ }^{7}$ and their development has been previously published ${ }^{8}$. Technologies selected for laboratory testing spanned four unique categories including: (i) pressure activated systems, (ii) pull tab alarms, (iii) audible alarms, and (iv) optically activated alarms.

The compilation of the master list of products was the result of a survey of the product literature, including sources such as the Thompson Register of products and internet searches. A total of 129 products fell into the overall category of devices capable of in-home surveillance of an individual.

Given the high cost, limited selection, and installation complexity of applicable surveillance technologies, we randomly selected 15 devices from all four categories for laboratory testing. However, device 15 was not operational and could not be used by the three engineers assigned to test it. Given that caregivers would also have difficulties with this device, this device was not tested.

The random selection procedure for the pull tab alarms sampled $21 \%$ of the offerings from the

Table 1. Sound pressure level and alarm audio frequency for each tested alarm product; n.a.=not applicable

\begin{tabular}{ccc|rccc}
\hline & Product & & \multicolumn{2}{c}{ Sound at distance, dB } & \multicolumn{2}{c}{ Alarm frequency, Hz } \\
\hline$\#$ & Type & Cost, US\$ & $\mathbf{0 . 1 5 m}$ & $\mathbf{1 2 m}$ & \multicolumn{1}{c}{ Minimum } & Maximum \\
\hline 1 & Floor mat & 158.25 & 99.5 & 45.7 & 697 & 1000 \\
2 & Floor mat & 141.90 & 104.3 & 69.0 & n/a & 2340 \\
3 & Floor mat & 151.75 & 96.5 & 52.4 & 2400 & 4900 \\
4 & Chair pad & 108.00 & 99.5 & 45.7 & 788 & 989 \\
5 & Chair pad & 80.00 & 119.0 & 64.8 & 1260 & 2900 \\
6 & Chair pad & 179.00 & 85.0 & 58.9 & 2100 & 2800 \\
7 & Bed pad & 66.50 & 99.5 & 45.7 & 788 & 989 \\
$8-$ Remote & Bed pad & 197.00 & 114.0 & 59.9 & 500 & 2500 \\
$8-$ Client & Bed pad & Included & 104.0 & 61.0 & n.a. & 2200 \\
9 & Bed pad & 229.00 & 85.0 & 58.9 & n.a. & 2150 \\
10 & Pull tab & 91.00 & 99.5 & 48.1 & n.a. & 700 \\
11 & Pull tab & 24.95 & 104.3 & 69.0 & n.a. & 2700 \\
12 & Pull tab & 45.95 & 109.7 & 75.1 & 2500 & 2800 \\
13 & Optical system & 80.75 & 85.2 & 52.0 & 600 & 800 \\
$14-$ Optical & Optical system & 39.00 & 87.6 & 57.9 & 550 & 690 \\
$14-$ Remote & Optical system & Included & 95.4 & 58.0 & 550 & 690 \\
\hline
\end{tabular}


master list, $23 \%$ of the audible alarms and $50 \%$ of the optically activated alarms and the pressure activated systems. The pull tab alarms consisted of 14 products, audible alarms of 13 products, and optically activated alarms of 6 products. A further 9 devices were selected from the pressure activated systems (18 products) which were subdivided further into floor mats (3), chair pads (3), and bed pads (3). Each device was ordered directly from the manufacturer or regional supplier in the USA. Devices ranged from US $\$ 20-U S \$ 800$ (Table 1).

\section{Protocols and variables}

Testing protocols for each device were devised to measure relevant operating parameters. While device designs varied, all emitted sound and were powered (either by battery or house current). Hence sound pressure level in $\mathrm{dB}$, alarm frequency (pitch), power consumption, and battery life were measured for each. These measures were obtained using the same protocol to facilitate direct comparisons. Other variables of interest (i.e. amount of force needed to release a pull tab) were specific to a given design and were gathered only for that device category (i.e. pull tab alarms). Because a technology in this theatre of prevention or monitoring assistance should meet a 'minimum set of criteria' in order to be the most effective, the measures below focus on capturing the technology's sound pressure levels, alarm frequency or pitch, power consumption, force measurements, and monitoring range, as applicable by device.

\section{Sound pressure levels}

The proper sound level may vary widely depending on many variables. A louder device may be more beneficial to caregivers who are suffering hearing loss and be some distance away from the actual device. However, loud devices may be disturbing to the person with dementia, increasing agitation and emotional upset.

Sound pressure levels of alarm intensity of each device were measured (in $\mathrm{dB}$ ) at multiple locations ranging from $0.15 \mathrm{~m}$ to $12 \mathrm{~m}$ using an Extech digital sound level meter (model 407768). The device was set at one end of the lab ( 12m away), the alarm activated and the sound level record. ed. Ambient decibel lev. el in the lab was $41 \mathrm{~dB}$.

Alarm frequency or pitch The frequency of an alarm tone is another important variable; the normal human ear is sensitive to frequencies

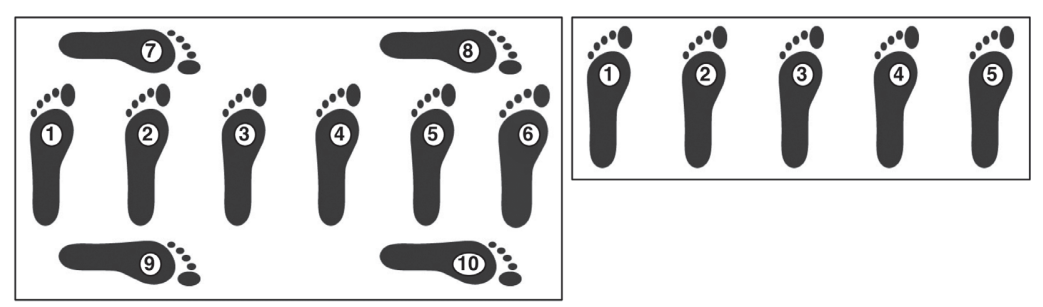

Figure 1. Diagram of foot placements for mat force testing ranging from 20-20,000 Hertz ( $\mathrm{Hz})$ with an optimum sensitivity at about $4,000 \mathrm{~Hz}^{8}$. However, with advancing age sensitivity to higher frequency sounds diminishes ${ }^{9}$. An alarm with a center frequency above $4000 \mathrm{~Hz}$ may not be perceptible by an aging caregiver who must be vigilant to alarm signals.

Frequency measurements were obtained via a microphone and a Tektronix TDS 2014 digital oscilloscope. Each alarm was activated and placed inside a soundproof box, measuring $610 \mathrm{~mm} x$ $406 \mathrm{~mm} \times 229 \mathrm{~mm}$, containing the microphone. The oscilloscope reported the frequency in $\mathrm{Hz}$.

\section{Power consumption}

All products tested used battery power to operate, although battery chargers allowing house current to substitute were available for some. Power consumption was measured in order to estimate battery life. Current draw in $\mathrm{mA}$ of each device was measured using a multimeter connected in series with a regulated power supply programmed to deliver power at manufacturer specified values. Measures of current were obtained under passive monitoring conditions and while the device was sounding its alarms (activated-low alarm, activated-high alarm). Reported values are the mean of three test measurements under each condition.

\section{Force measurements}

The force and pressure required to activate the audible alarm for the floor mats were measured and calculated, respectively. A size 13 shoe of medium width was used as the surface contact interface to each mat. A Chattilon force gauge (range: $0-1000 \mathrm{~N}$; dial resolution: $5 \mathrm{~N}$; accuracy limits: +/- $0.5 \%$ of full scale) was used to relay force through the shoe. A metal bar was used as a stiffening member within the shoe to evenly distribute the applied load. The force was gradually increased to the shoe system until the alarm sounded. When the alarm was activated the force reading was recorded. This force measuring system was used at various locations (Figure 1) on the mat to observe force variance. Each force measurement was repeated three times at each location and the mean was calculated. 
From the measured force data, calculations were made to determine the pressure required for device activation. Inter-location variability estimates are presented.

Only one foot was needed to obtain the correct surface area and apply uniform pressure to the mat for the standard foot size. Mats of different sizes were tested; the mat on the right (Figure 1) was tested using the foot arrayed in parallel, which is the likely configuration for this mat if placed at the foot of a bed. The left (larger) mat of Figure 1 could be rotated and the foot was applied in two directions.

Force measurements of chair pads were done in a different way. These devices sound an alarm when an internal sensor detects a change in force on the chair pad and are important to alert a caregiver that the monitored individual has left the chair and may require immediate supervision. To measure the activation force applied to the pressure sensitive chair pad, a semi-circle aluminum plate, simulating the posterior surface of a $75^{\text {th }}$ percentile US adult male, was used as the human simulant. A Chattilon force gauge for larger force measurements $(>90 \mathrm{~N})$ and a ComTen digital force gauge for smaller force measurements $(<90 \mathrm{~N})$ were used to apply force to the pad. The force was gradually increased on the plate until the force required to activate the chair pad was obtained. Then the force was slowly reduced until the alarm sounded and the force measurement was recorded. The process was repeated three times and the mean calculated.

Force measurements for pressure sensitive bed pads were made on a standard hospital bed (Hill-Rom Inc.). The pad was placed beneath a sheet in the middle of the mattress. A flat plate measuring $0.3 \mathrm{~m} \times 0.2 \mathrm{~m}$ was used to evenly distribute the force over the pad. A Chattilon force gauge was used to apply force to the pad. The force was gradually increased to the plate until the device reached its 'activation force' and was armed. The force was slowly reduced until the alarm sounded which was termed the device's 'release force'. When the alarm sounded the force reading at that time was recorded. Each force measurement was repeated three times and the mean force was calculated.

Pull tab alarms are generally used to alert the caregiver that the individual is arising from a chair. The force required to sound the alarm for each pull tab device was measured. A Com-Ten digital force gauge attached to the device's pull cord applied tension until the alarm sounded; this force, termed 'magnet pull force', was recorded. The force required to pull the clip end of the pull cord free from a standard hospital garment was also measured. The clip was attached to a loose fitting hospital garment on a mannequin at two test locations; the hem, and the collar, which was selected because its thickness made it more resistant to tearing. The digital force gauge was then used to pull the clip free from the clothing (clip pull force - clothes) and free from the collar (clip pull force - collar). Each force measurement was taken three times and the mean calculated.

\section{Activation area}

Optically activated alarms send a discreet chime to the receiver up to 100 feet $[30.48 \mathrm{~m}]$ away or allow the care receiver to pre-record a 10s word message. Whenever anyone approaches, the message is announced. It will continue to repeat itself until the person leaves the talking sign range. These systems were composed of two units, a motion sensor and a remote alarm. These can be used to determine when the person with dementia gets out of bed. In that instance this person will break the beam and the motion sensor will send a message to the remote alarm or enters the range of the alarm. The area in which the device was activated and the alarm triggered was measured and consisted of two distinct tests:

(i) Direct approach to device at angles of $0^{\circ}, 45^{\circ}$, $90^{\circ}, 135^{\circ}$, and $180^{\circ}$ (Figure 2) that were mapped out on the floor relative to the optical sensor (positioned at $90^{\circ}$ ) and the investigator walked towards the device precisely along the angle lines. The distance from the device when the alarm was activated was recorded. This yielded a relative sensitivity
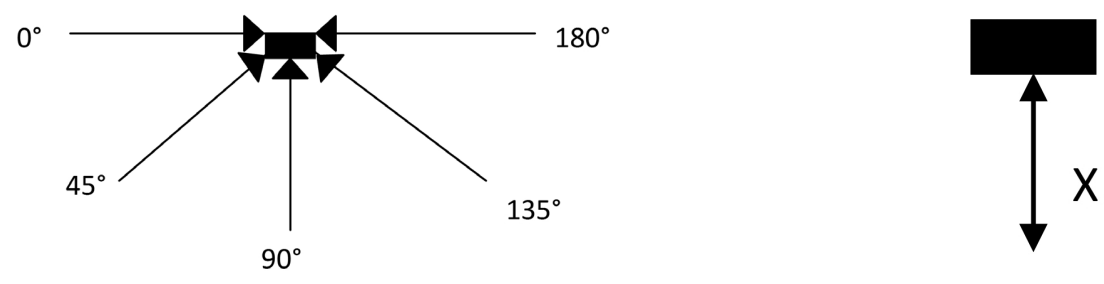

Figure 2. Diagram for direct approach testing of optically activated alarms

Figure 3. Diagram for parallel pass testing of optically activated alarms; $X$ is the distance in which the device was activated 
map of the device as a function of approach vector. (ii) Parallel pass-by of device approach. Here the investigator walked parallel to the device and at right angles to the field of view of the optical sensor. The distance $(X)$ in which the alarm was activated was recorded. A 2D plan view of the device activation area was mapped by combining these two approaches. Each device was installed at a height of five feet $[1.52 \mathrm{~m}]$ above the floor (Figure 3).

\section{RESULTS}

Alarm sound pressure levels varied widely depending on the device (Table 1). Many devices had multiple alarm tones and volume settings; only the highest $\mathrm{dB}$ values for each alarm are reported as boundary conditions for the device. All other values for each device fall between these two demarcations. For comparison, $45 \mathrm{~dB}$ is a normal sound to awaken a sleeping individual, $60 \mathrm{~dB}$ is normal conversation, and $100 \mathrm{~dB}$ is sound from a car horn at 5 meters.

Some devices have a range of frequencies as the alarm altered its pitch through the alarm sequence (Table 1). In these cases both the minimum and maximum values are reported. Product 20 could not be tested as all the electrical components were factory sealed and opening the case would have damaged the device.

The greater the power consumption of a device the quicker the batteries will be drained. The power requirements of a device can vary depending on how the device is used. Standard alkaline batteries were used and testing was based on the product's intended use. Lab test results are given in three categories (Table 2):

(i) Power consumption with minimal load (device is on and monitoring but no alarm is activated); (ii) Power consumption with low alarm activating (lowest alarm setting on device);

(iii) Power consumption with loudest alarm activating (highest alarm setting on device when applicable).

In addition, calculations were made to estimate battery life for each device. Battery life can be difficult to estimate as many variables can either extend or shorten the overall life. Therefore, these estimations can be either longer or shorter than what actually may be experienced. These estimations are made to offer a comparison of devices acting under identical electrical loading conditions. To estimate the battery life for each device the following assumptions were made: (i) Device would be on 24 hours a day and in the monitoring mode (minimal load) when the alarm was not activated, and (ii) Device would be activated using its highest alarm mode (greatest current draw) for 5 minutes every hour over a 12 hour period. This time frame would correspond to a total of 60 minutes every day.

Force measurement data (Table 3) for pressure activated systems and pull tab alarms showed a great variation. Products $6(\mathrm{CP})$ and 9 (BP) could be set to any threshold activation force and could not be tested. Products 10 (PT) and 11 (PT) clip attachment would not release from the collar without ripping the mannequin's garment.

Table 2. Power consumption assessment for each tested alarm product; n.a.=not applicable

\begin{tabular}{ccc|rccc}
\hline & Product & & \multicolumn{2}{c}{ Sound at distance, dB } & \multicolumn{2}{c}{ Alarm frequency, Hz } \\
\hline$\#$ & Type & Cost, US\$ & $\mathbf{0 . 1 5 m}$ & $\mathbf{1 2 m}$ & \multicolumn{1}{c}{ Minimum } & Maximum \\
\hline 1 & Floor mat & 158.25 & 99.5 & 45.7 & 697 & 1000 \\
2 & Floor mat & 141.90 & 104.3 & 69.0 & n/a & 2340 \\
3 & Floor mat & 151.75 & 96.5 & 52.4 & 2400 & 4900 \\
4 & Chair pad & 108.00 & 99.5 & 45.7 & 788 & 989 \\
5 & Chair pad & 80.00 & 119.0 & 64.8 & 1260 & 2900 \\
6 & Chair pad & 179.00 & 85.0 & 58.9 & 2100 & 2800 \\
7 & Bed pad & 66.50 & 99.5 & 45.7 & 788 & 989 \\
$8-$ Remote & Bed pad & 197.00 & 114.0 & 59.9 & 500 & 2500 \\
$8-$ Client & Bed pad & Included & 104.0 & 61.0 & n.a. & 2200 \\
9 & Bed pad & 229.00 & 85.0 & 58.9 & n.a. & 2150 \\
10 & Pull tab & 91.00 & 99.5 & 48.1 & n.a. & 700 \\
11 & Pull tab & 24.95 & 104.3 & 69.0 & n.a. & 2700 \\
12 & Pull tab & 45.95 & 109.7 & 75.1 & 2500 & 2800 \\
13 & Optical system & 80.75 & 85.2 & 52.0 & 600 & 800 \\
$14-$ Optical & Optical system & 39.00 & 87.6 & 57.9 & 550 & 690 \\
$14-$ Remote & Optical system & Included & 95.4 & 58.0 & 550 & 690 \\
\hline
\end{tabular}


Table 3. Force measurement data of each of the relevant tested alarm products; n.a. $=$ not applicable

\begin{tabular}{|c|c|c|c|c|c|}
\hline \multicolumn{2}{|r|}{ Product } & \multirow{2}{*}{$\begin{array}{c}\text { Mean activation, } \\
\mathrm{N}\end{array}$} & \multirow{2}{*}{$\begin{array}{l}\text { Magnetic sensor } \\
\text { pull, } \mathrm{N}\end{array}$} & \multirow{2}{*}{$\begin{array}{l}\text { Clip pull on } \\
\text { clothes, } N\end{array}$} & \multirow{2}{*}{$\begin{array}{c}\text { Clip pull on } \\
\text { collar, } N\end{array}$} \\
\hline$\#$ & Type & & & & \\
\hline 1 & Floor mat & 18.82 & n.a. & n.a. & n.a. \\
\hline 2 & Floor mat & 27.98 & n.a. & n.a. & n.a. \\
\hline 3 & Floor mat & 29.67 & n.a. & n.a. & n.a. \\
\hline 4 & Chair pad & 73.88 & n.a. & n.a. & n.a. \\
\hline 5 & Chair pad & 8.36 & n.a. & n.a. & n.a. \\
\hline 6 & Chair pad & Varies & n.a. & n.a. & n.a. \\
\hline 7 & Bed pad & 46.13 & n.a. & n.a. & n.a. \\
\hline 8 & Bed pad & 31.09 & n.a. & n.a. & n.a. \\
\hline 9 & Bed pad & Varies & n.a. & n.a. & n.a. \\
\hline 10 & Pull tab & n.a. & 4.48 & 24.26 & Remained on \\
\hline 11 & Pull tab & n.a. & 6.11 & 16.13 & Remained on \\
\hline 12 & Pull tab & n.a. & 1.78 & 9.73 & 28.94 \\
\hline
\end{tabular}

The activation area for the optically activated systems showed variation as well (Figure 4). In some instances the device failed to activate on a direct approach. Conversely, the laboratory space was inadequate to cover the device activation range at over $18 \mathrm{~m}$ resulting in an inability to accurately assess the 45 and 135 degree angles. Product 14 had two methods of attaching the optical sensor ('level' and 'survey views') and thus produced two sets of data. Level view has the device installed horizontal and thus using the longer dimension for optical sensor (2.0inches; $50 \mathrm{~mm}$ ). Survey view has the device installed vertically and thus using the shorter dimension for the optical sensor (1.13inches; 287mm).

\section{Discussion}

This study conducted a systematic examination of commercially available technologies that may be useful to caregivers to continuously monitor a person with dementia at risk for a missing incident from the home. This study found that none of the randomly selected commercially available technologies tested were effective in preventing a missing incident by supporting the needed surveillance of a person with dementia. The products should have met a 'minimum set of criteria' in order to be the most effective. All of the products tested in this laboratory evaluation failed in at least one criterion that was deemed significant (for instance, sound pressure levels, alarm frequency or pitch, power consumption, force measurements, and monitoring range).

Products alerting caregivers to motion in the home were pressure sensitive floor mats, chair pads, bed pads, as well as pull tab, audible and optically activated alarms. For these products to be useful in a home set- ting they must not have a loud alarm at the site of the person with dementia to avoid agitating or scaring them but be loud enough and have an adequate frequency so that they can be heard by the caregiver. The products should be as power efficient as possible to alleviate the need to constantly replace batteries. The products should have a remote alarm at the site of the caregiver, and must be able to account for unusual patterns of activity accurately and reliably. Sensitivity is very important as the device needs to activate when required (true positive) but not overly sensitive to activate erroneously (false positive, false negative).

None of the products studied met the minimum set of criteria stated above. While most of the products had a suitable alarm frequency, there were 3 products (products 5, 17, 8a ) that had sound pressure levels above $110 \mathrm{~dB}$, or sound close to a train, at a distance of $0.15 \mathrm{~m}$. A loud alarm of this type would be severely agitating to anyone, especially a person suffering with dementia. Of the products tested in this group, there were only 2 (products 8,14 ) that had a remote alarm that
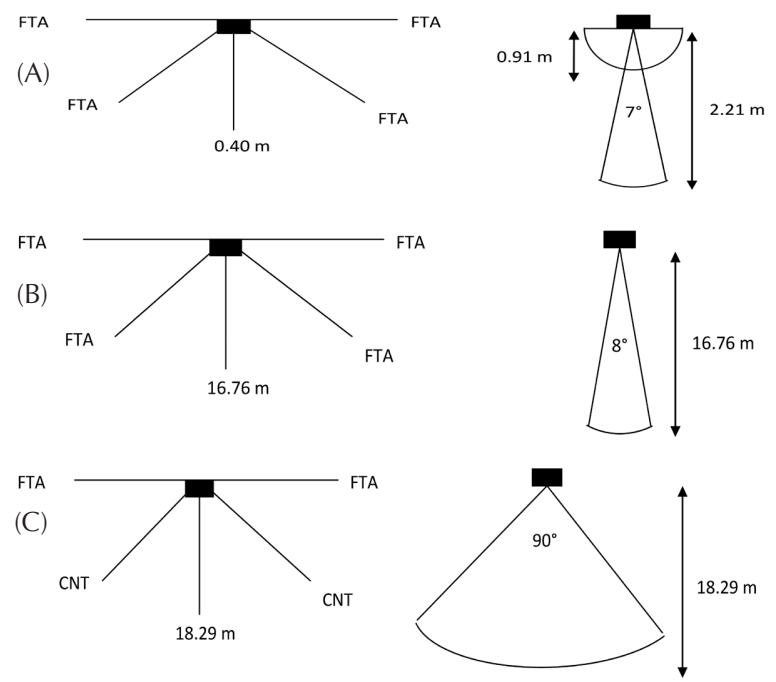

Figure 4. Area maps with activation distances; $A=$ product 13; $B=$ product 14 in 'survey' view; $C=$ product 14 in 'level' view; FTA=failed to activate; $C N T=$ could not be tested 


\section{Commercial allarm products}

could be taken anywhere in a home with a caregiver but with an extremely loud sound.

In simulated power consumption, most devices would require new batteries in less than 20 days. A majority of the devices tested were rated as using only modest power estimation requirements, but only five products (Products 3, 10, 11, 13, 16) were able to have battery life last more than three weeks.

Sensitivity fluctuated among the devices with the pressure activated products having the most disparity. Two of the pressure activated systems (Products 6, 9) had a user set threshold for device activation. This feature should prove most helpful as a caregiver can set their personal level of sensitivity for monitoring. Conversely, there were multiple pressure activated products (Products 1 , $2,3,5)$ that required less than $30 \mathrm{~N}$ of force to activate the alarm. This low force threshold means that the majority of true positives will cause correct alarms but also a multitude of false positives will cause a false alarm. This fact is especially true for floor mats, audible alarms and optical alarms as these devices have no ability to discern the person with dementia from the caregiver or anyone else in the house, including a family pet.

\section{Conclusion}

Older adults prefer to live independently and age in place. However, caregivers of persons with dementia are challenged with the stress and burden of providing 24/7 surveillance and care, increasing the risk for premature nursing home placement of an older adult with dementia. The pressure activated systems, pull tab alarms, audible alarms, and optically activated alarms tested here are important because these commercially available products may be used to help a caregiver better care for a person with dementia and relieve some of the stress and burden associated with constant surveillance. However, practical issues, such as a 'minimum set of criteria', must be considered before these technologies are recommended for implementation in the home environment.

The technologies tested in this investigation spanned a wide array of surveillance categories. This laboratory investigation is the first step in assessing the merits of various types of tech-

\section{Acknowledgements}

The work presented here was supported by grants from the VA VISN 8 Patient Safety Center of Inquiry, Tampa, FL and the James A. Haley VA Hospital, Tampa, FL. Portions of this study were presented at the "9th Annual Transforming Fall Prevention nologies that may be used to keep the person with dementia safe in the home and prevent missing incidents from the home with continuous surveillance. The best technology for these purposes is one that is tailored to the unique circumstances of the individual caregiver and care recipient and which provides the most benefit to each. Future steps need to be taken to investigate how these devices perform outside the lab in the actual setting that they are intended (home, institution) and to develop a standardized method of testing in order to directly compare products.

The findings from this research suggest that developers interested in such applications should consider adequate sound pressure levels, alarm frequency or pitch, power consumption, force measurements, and monitoring range for their devices to be most useful to caregivers of persons with dementia. Caregivers, in turn, will be more willing to adopt these devices when they can reliably ensure that these technologies are an acceptable surveillance intervention that may be used to effectively keep the care receiver safe. There are likely other factors for developers to consider that we could not test here in this lab study. For example, though floor mats are useful in providing location information and movement to a caregiver, preliminary research shows that these mats may also be a trip hazard to some older adults in the home ${ }^{10}$. Other devices may not work because they have to be constantly refitted, for example, there is some evidence that chair pads have to be continuously readjusted to work and be comfortable. Additional work in the home environment is needed to test the practicality and usability of each of these devices even after the 'minimum set criteria' is met.

Though tested singly here, it is recognized that a single technology will not be the total solution to diminish or prevent missing incidents. Rather, the use of multiple technologies working in concert and with regular caregiver supervision is undoubtedly the best hindrance to a missing incident. However, this strategy is quite cumbersome, especially for a family caregiver with low educational attainment and/or little experience using some of these technologies. Additional work is needed to determine how these technologies may be linked into a single system to meet the caregiver and care recipient's needs.

Practices" conference in Clearwater, FL in 2008. We would like to thank James Fozard, PhD and Michael Kerrigan, MS for their helpful comments and suggestions on this manuscript. The authors' views are their own and do not represent those of the Department of Veterans Affairs. 


\section{Commerciall allem products}

\section{References}

1. Rowe MA, Vandeveer SS, Greenblum CA, List CN, Fernandez RM, Mixson NE, Ahn HC. Persons with dementia missing in the community: Is it wandering or something unique? BMC Geriatrics 2011;11(28):1-8; doi:10.1186/1471-2318-11-28

2. Bowen ME, McKenzie B, Steis M, Rowe MA. Prevalence of and antecedents to dementia-related missing incidents in the community. Dementia and Geriatric Cognitive Disorders 2011;31(6):406412; doi:10.1159/000329792

3. Miyamoto Y, Hiroto I, Otsuka T, Kurita H. Caregiver burden in mobile and non-mobile demented patients: a comparative study. International Journal of Geriatric Psychiatry 2002;17(8):765-773; doi:10.1002/gps.694

4. Creese J, Bédard M, Brazil K, Chambers L. Sleep disturbances in spousal caregivers of individuals with Alzheimer's disease. International Psychogeriatrics 2008;20(1):149-161; doi:10.1017/ S1041610207005339

5. McShane R, Gedling K, Keene J, Fairburn C, Jacoby R, Hope T. Getting lost in dementia: a longitudinal study of a behavioral symptom. International Psychogeriatrics 1998;10(3):253-260; doi:10.1017/S1041610298005365
6. Rowe MA, Fehrenbach N. Injuries sustained by community-dwelling individuals with dementia. Clinical Nursing Research 2004;13(2):98-110; doi:10.1177/1054773803262520.

7. Algase DL, Kupferschmid B, Beel-Bates C, Beattie E. Estimates of Stability of Daily Wandering Behavior Among Cognitively Impaired Long-Term Care Residents. Nursing Research 1997;46(3):172-178

8. Kearns W, Rosenberg D, West L, Applegarth S. Attitudes and expectations of technologies to manage wandering behavior in persons with dementia. Gerontechnology 2007;6(2):89-101; doi:10.4017/ gt.2007.06.02.004.00

9. Nave CR. Frequencies for Maximum Sensitivity of Human Hearing. 2005; http://hyperphysics.phyastr.gsu.edu/Hbase/sound/maxsens.html; retrieved January 15, 2012

10. Fozard J, Gordon-Salant S. Changes in vision and hearing with aging. In: Birren J, Schaie K, editors, Handbook of the Psychology of Aging, 5th edition. San Diego: Academic Press; 2001; pp 241-266

11. Skubic M, Alexander G, Popescu M, Rantz M, Keller J. A Smarthome Application to Elder Care: Current Status and Lessons Learned. Technology and Health Care 2009;17(3):183-201; doi:10.3233/ THC-2009-0551 